\title{
La melatonina no es eficaz para mejorar el insomnio secundario ni los trastornos por deprivación de sueño
}

Buscemi N y col. BMJ 2006; 332:385-93.

\begin{abstract}
Objetivo
Evaluar la eficacia y seguridad de la melatonina exógena en desórdenes secundarios del sueño y en trastornos relacionados con la restricción de horas de sueño, tal como el "jet lag" (asociado a un viaje en avión a través de 2 husos horarios diferentes) o desórdenes relacionados con horarios rotativos de trabajo.

\section{Fuentes de datos}

Se revisaron 13 bases de datos electrónicas (que incluyeron MEDLINE, EMBASE y Cochrane) así como revisión manual de resúmenes relevantes de la Asociación de Profesionales de la Sociedad del Sueño (año 1999 a 2003).

\section{Selección de estudios}

Se incluyeron: a) ensayos clínicos controlados y aleatorizados que evaluaran la eficacia de la melatonina comparada con placebo con relación a algún resultado principal, como latencia al sueño, tiempo total de sueño, y calidad del mismo; y b) ensayos clínicos aleatorizados y no aleatorizados que reportaran eventos adversos para evaluar la seguridad. Los pacientes incluidos debían padecer un trastorno secundario del sueño (debido a una enfermedad médica o psiquiátrica crónica) o padecer restricción del sueño (relacionada con viajes aéreos o por horarios de trabajo rotativos). Los estudios fueron evaluados de acuerdo a su calidad metodológica utilizando varias escalas validadas por dos revisores independientes.
\end{abstract}

\section{Resultados principales}

Se incluyeron 12 estudios de insomnio secundario y 13 de deprivación de sueño. La calidad metodológica fue buena en la mayoría de los trabajos. Seis trabajos (97 participantes en total) no mostraron evidencia de eficacia de la melatonina en insomnio secundario (con una diferencia media ponderada* (DMP) no significativa de 13,2 minutos en la latencia del sueño con melatonina en relación al placebo (IC95\% -27,3 a 0,9). Nueve estudios (427 participantes) tampoco evidenciaron eficacia de esta droga en personas con desórdenes por restricción de sueño: DMP en latencia de sueño de 1 minuto a favor de la melatonina (IC95\% -2,3 a 0,3 minutos). Diecisiete estudios (651 participantes) no encontraron mayor frecuencia de efectos adversos de la melatonina con relación al placebo, usada por corto tiempo (tres meses o menos).

\section{Conclusiones}

No se encontró evidencia de que la melatonina sea efectiva en desórdenes secundarios de sueño o desórdenes asociados a restricción de sueño.

Fuente de financiamiento: Instituto Nacional de Salud Canadiense y varias fundaciones hospitalarias y privadas.

\section{Comentario}

En septiembre de 2005, el Instituto Nacional de Salud de Estados Unidos publicó que 70 millones de personas de ese país sufrían algún trastorno de sueño, de los cuales el 59\% tenían desórdenes crónicos.

Cada año 100.000 accidentes se asocian a quedarse dormido mientras se conduce. La privación de sueño y la excesiva somnolencia diurna cuestan alrededor 16 mil millones de dólares anuales a los EEUU'. En Argentina y nuestra región no contamos con estadísticas relacionadas a con este tema, pero podríamos extrapolar a nuestra realidad los datos arriba mencionados para tomar conciencia de la magnitud del problema.

En el caso de insomnio son muchas las medicinas alternativas que se utilizan, inclusive de venta libre, y una de las más conocidas es la melatonina.

Esta hormona, naturalmente secretada por la glándula pineal, y que se encuentra ligada al ritmo circadiano, se administra de modo externo en dosis mínimas, en un intento de "restaurar" el ritmo circadiano en desórdenes del sueño exógenos ${ }^{2}$ (como insomnio secundario o relacionado a deprivación de sueño).

Los autores del artículo concluyeron que la melatonina exógena no es útil para el tratamiento de este tipo de desórdenes. No obstante aclaran que no se determinó la eficacia de esta droga en lo que respecta a fatiga diurna y mejoría de la calidad de vida, por lo que los resultados no se contradicen con los de dos revisiones sistemáticas previas ${ }^{3,4}$, en donde la melatonina resultó útil para aliviar síntomas de fatiga diurna relacionados al "jet lag".

Si bien la melatonina no parece eficaz para mejorar los parámetros relacionados al sueño se ha desarrollado un nuevo análogo sintético, aprobado el año pasado por la Administración de Drogas y Alimentos de USA (FDA), el ramelteon, que actuaría como agonista de los receptores MT1, atenuando la señal de alerta del núcleo supraquiasmático, promocionando el sueño, como así también sobre MT2 sincronizando el reloj circadiano. Este medicamento aún no ha ingresado a nuestro país, aunque se sabe que ocasiona mareos, incremento de los niveles de prolactina, y supuestamente disminuye el tiempo de latencia del sueño ${ }^{5}$.

Faltan, de todas maneras, datos provenientes de estudios aleatorizados que demuestren la eficacia de este promisorio fármaco.

\section{Conclusiones de la comentadora}

La melatonina no es efectiva para mejorar los desórdenes secundarios de sueño o los asociados a restricción del mismo, aunque sí existen datos de eficacia para aliviar los síntomas de fatiga diurna relacionados al "jet lag". Sería deseable continuar investigando otros efectos relacionados con la melatonina, ya que por ejemplo un trabajo demostró que aquellos pacientes con Alzheimer que tomaban melatonina evidenciaban menor deterioro cerebral que aquellos que no la recibían ${ }^{6}$.

Valiensi S. La melatonina no es eficaz para mejorar el insomnio secundario ni los trastornos por deprivación de sueño. Evid. actual. práct. ambul. 9(5):140. SeptOct.2006. Comentado de: Buscemi, N, Vandermeer, B, Otón, N et al.Efficacy and safety of exogenous melatonin for secondary sleep disorders and sleep disorders accompanying sleep restriction: meta-analysis. BMJ 2006 Feb 18;332(7538):385-93.PMID:16473858.

Nota para los más interesados: en Argentina trabaja el Dr. Daniel Cardinali, excelente investigador del tema, y para aquellos que deseen más información pueden consultar su página web en www.daniel-cardinali.medem.com y enterarse de los últimos avances.

\section{Bibliografía}

1. Buysse D. Current perspectives in insomnia, Medscape. 23 september 2005.vol. 6 CME.

2. The international classification of sleep disorders: diagnostic \& coding manual, ICDS-2. 2nd. Ed. Westchester, III: American Academy of Sleep Medicine, 2005

3. Herxheimer A, Petrie KJ. Melatonin for preventing and treating jet lag. Cochrane Database Syst Rev 2002;2: CD001520.

4. Chase JE, Gidal BE. Melatonin: therapeutic use in sleep disorders. Ann Pharmacother 1997;31: 1218-26.

5. NIH research on Sleep and Sleep disorders Program Announcement (2-9-2005).

6. Silber M. et al. Chronic Insomnia.N ENgl J Med 353, 8. August 25, 2005; 803-809. 